\title{
Scorpion venom peptide, BmK AGAP as an adjuvant to lidocaine in sciatic nerve block causes dose- dependent increase intensity of sensory block and prolog duration of analgesia in rat.
}

Sylvanus Kampo

Dalian Medical University

Tingting Zhou RO

Dalian Medical University

Qing-Ping Wen ( $\nabla$ sylvanus.kampo@uds.edu.gh )

Dalian Medical University https://orcid.org/0000-0002-9700-7975

\section{Research Article}

Keywords: Scorpion venom peptide, BmK AGAP, nerve block, adjuvant, perioperative analgesia

Posted Date: January 4th, 2019

DOI: https://doi.org/10.21203/rs.2.176/v1

License: (c) (1) This work is licensed under a Creative Commons Attribution 4.0 International License.

Read Full License 


\section{Abstract}

Background: Various analgesics adjuvants, including opioids, have been tested and demonstrated to be clinically beneficial when added to local anesthetics in an attempt to increase the duration of analgesia with the risk of various adverse effects. This study was designed to test the hypothesis that scorpion venom peptide, BmK AGAP as an adjuvant to lidocaine causes dose-dependent increase intensity of sensory block and prolong duration of analgesia.

Method: We performed partial sciatic nerve ligation on 60 rats to induce a rapid onset and long-lasting mechanical allodynia. Equal volume $(600 \mu \mathrm{l})$ of preservative-free lidocaine $(0.5 \%)$ and BmK AGAP (2 or 1 $\mathrm{mg} / \mathrm{kg}$ ) were prepared with saline $(0.9 \%)$. The rats were randomly allocated into five groups. Group A $(n=12)$ received saline as the negative control; Group $B(n=12)$ received equal volume of lidocaine and saline; Group C $(\mathrm{n}=12)$ received lidocaine and BmK AGAP (1 mg/kg); Group D ( $\mathrm{n}=12)$ received lidocaine along with BmK AGAP $(2 \mathrm{mg} / \mathrm{kg})$ and Group $E(n=12)$ sham. We operated on the rats representing the sham group $(n=12)$ and the sciatic nerve exposed but not ligated. The von Frey filaments were used to assessed mechanical allodynia in rats.

Results: The data showed that rats from the lidocaine group exhibited increased $12.51 \mathrm{~g}$ of PWT. The rats from the BmK AGAP (1 mg/kg) group exhibited increased $13.67 \mathrm{~g}$ of PWT, whereas the rats from the BmK AGAP $(2 \mathrm{mg} / \mathrm{kg})$ group exhibited increased $14.78 \mathrm{~g}$ of PWT compared with the baseline PWT. There was a prolonged duration of increased paw withdrawal threshold in rats that were supplemented with BmK AGAP compared with rats that received lidocaine injection alone. We realized a significant $(P<0.0001)$ prolonged duration of increased paw withdrawal threshold in rats that were supplemented with $B m K$ $\operatorname{AGAP}(2 \mathrm{mg} / \mathrm{kg})$ compared with rats that received BmK AGAP $(1 \mathrm{mg} / \mathrm{kg})$.

Conclusion: This study finding suggests scorpion venom peptide, BmK AGAP to be a potent analgesic adjuvant. BmK AGAP as an adjuvant to lidocaine in sciatic nerve block increase the intensity of sensory block and prolongs perioperative analgesia without significant adverse effects.

Keywords: Scorpion venom peptide, BmK AGAP, nerve block, adjuvant, perioperative analgesia

\section{Background}

A nerve block is achieved by administering local anesthetics to a nerve to numb a particular region of the body to facility surgery or manage pain. The benefits of a peripheral nerve block include adequate pain control and less general anesthesia-related side effects. However, the fear of possible failed blocks and undesirable effects still limits the acceptance of these techniques. For the past years, efforts have been made to minimize these unwanted events and optimize the patient experience. The addition of analgesic adjuvants to local anesthetic preparations to achieve adequate pain relief while reducing the total dose of local anesthetic has been one of the options followed to realize these goals [1, 2]. 
Numerous analgesics have been tested and demonstrated to be clinically beneficial when added to local anesthetics for peripheral nerve blocks or when used for local infiltration. Analgesics such as morphine, fentanyl, buprenorphine, and tramadol when added to local anesthetics reduce the total required dose of the local anesthetic, prolong sensory block, minimize central nervous system effects, and enhance postoperative analgesia [2]. However, study reports show that the use of these adjuncts is limited because they cause dose-dependent pruritis, urinary retention, and nausea. These suggest the need for new supplements that will provide faster recovery without compromising anesthetic reliability.

The scorpion Buthus martensii Karsch venom and its extracts are used to treat pain. In 1994, the first BmK analgesic peptide was purified from the toxin [3]. Since then, more analgesic peptides including BmK AGAP (Buthus martensii Karsch Analgesic-Antitumor Peptide) are purified for pain. Liu Y-F et al., in 2002 demonstrated the analgesic activity of BmK AGAP through the intraperitoneal injection in mice. It was realized that BmK AGAP exhibits a strong analgesic activity compared with morphine [4]. Li et al. in 2014 demonstrated the antinociceptive effect of BmK AGAP through the tail vein injection and realized a similar strong analgesic activity compared with morphine [5]. Ruan et al., in 2018 reported that intrathecal injection of the Chinese scorpion venom peptide BmK AGAP inhibits neuropathic and inflammationassociated pain through a MARK-mediated mechanism [6]. Authors of this study hypothesized that BmK AGAP as an analgesic adjuvant to lidocaine in sciatic nerve block causes dose-dependent increase intensity of sensory block and prolong duration of perioperative analgesia. Hence, we aimed to investigate the effects of BmK AGAP along with lidocaine in a rat model of sciatic nerve blockade.

\section{Methods}

\section{Drugs}

Preservative-free $2 \%$ lidocaine (31802232) was purchased from the northeast pharmaceutical group (China).

\section{Preparation of recombinant BmK AGAP}

The recombinant BmK AGAP (rBmK AGAP) for this study was provided by the Shenyang Pharmaceutical University School of Life Science and Bio-pharmaceutics (Shenyang, China). The process of obtaining the rBmK AGAP was the same as previously described [7]. The activity of rBmK AGAP was the same as the previous.

\section{Animals}

Adult (6 weeks) Sprague Dawley male rats weighing between 180 and $200 \mathrm{~g}$ were purchased from the animal facility of Dalian Medical University for this experiment. The rats were housed in standard transparent plastic cages under 12-hours/12-hours light-dark cycle regime and were provided free access to food and water. The Dalian Medical University, animal ethics committee, approved this prospective 
randomized, double-blind study and it was by the declaration of the national institutes of health guide for the care and use of laboratory animals.

\section{Partial sciatic nerve ligation (PSL)}

The procedure used for the partial sciatic nerve ligation (PSL) model was mainly the same as that reported by Seltzer and Bennett $[8,9]$. The rats were anesthetized with sodium pentobarbital $(40 \mathrm{mg} / \mathrm{kg}$, intraperitoneal injection) and a small incision made at the mid-thigh level to expose the right sciatic nerve. A unilateral tight ligation of one-third of the sciatic nerve was induced with a single ligature (5 - 0 silk thread). We operated on the rats representing the sham group and the sciatic nerve exposed but not ligated. The incision made was closed in layers and the surgical wound treated with antibiotics (Vetericyn Plus antimicrobial hydrogel) daily. We confirmed the presence of mechanical allodynia in rats, second day after PSL. Rats were then randomly assigned to one of five groups using a computer-generated random number table. The group allocation was concealed in a sealed opaque envelops which were opened just before administration of the block. Group $A(n=12)$ received $0.9 \%$ saline as the negative control; Group B ( $\mathrm{n}=12)$ received equal volume of $0.5 \%$ lidocaine $(4 \mathrm{mg} / \mathrm{kg})$ along with $0.9 \%$ saline; Group $C(n=12)$ received $0.5 \%$ lidocaine $(4 \mathrm{mg} / \mathrm{kg})$ along with BmK AGAP $1 \mathrm{mg} / \mathrm{kg}$; Group D $(\mathrm{n}=12)$ received $0.5 \%$ lidocaine $(4 \mathrm{mg} / \mathrm{kg})$ along with BmK AGAP $2 \mathrm{mg} / \mathrm{kg}$, and Group $\mathrm{E}(\mathrm{n}=12)$ the sham (rats were operated and the sciatic nerve exposed but not ligated).

\section{Drugs Application.}

The equal volume of drugs solutions (preservative-free $0.5 \%$ lidocaine $(4 \mathrm{mg} / \mathrm{kg}$ ) and BmK AGAP 2 or $1 \mathrm{mg} / \mathrm{kg}$ ) were prepared by an anesthesiologist not involved in the data collection and administered as a single injection of $0.5 \mu \mathrm{L} / \mathrm{kg}$ up to a maximum of $600 \mu \mathrm{L}$. An independently specialized anesthesiologist in regional anesthesia was assigned to perform the sciatic nerve block. Three independent researchers specialized in pain assessment and were blinded to drugs administration were assigned to assess sensory block, motor block, and mechanical allodynia after drugs administration. The doses of the drugs were determined based on previous studies $[4,5,10]$. The sciatic nerve in the right hind was located in the upper-thigh level using a nerve stimulator (Stimpod NMS450, Emergo Europe, and the Netherlands). A 22gauge short bevel electrically insulated electrode (AB-22025-SS) was advanced through the gastrocnemius and biceps femoris muscles. The motor response elicited by $0.2-0.5 \mathrm{~mA}$ (flicking) confirmed the location of the sciatic nerve and the appropriate position of the electrically insulated electrode. $0.5 \%$ lidocaine $(4 \mathrm{mg} / \mathrm{kg}$ ) was then injected under the nerve simulator guidance. An equal volume $(600 \mu \mathrm{l})$ of $0.9 \%$ saline or BmK, AGAP 2 or $1 \mathrm{mg} / \mathrm{kg}$ was added to lidocaine injection. The block performance time (time elapsed from electrically insulated electrode positioning to the end of local anesthetic injection) and number of time attempts were recorded. Complications, including vascular puncture, were noted. A successful block was defined as sensory blockade in all regions assessed within 30 minutes of local anesthetic injections. Duration of analgesia was defined as the time interval between the administration of drugs solutions and complete resolution of anesthesia. Motor block (time since the 
administration of the block until the absence of movement in the right hind). Rats that exhibited complete motor block were excluded from this study.

\section{Measurement of mechanical allodynia}

The von Frey filaments (North Coast Medical, Inc., San Jose, CA) was used to assess the mechanical allodynia after the drugs were injected. The starting point of the filaments used was from $2 \mathrm{~g}$ and ended with $0.16 \mathrm{~g}$ or $15 \mathrm{~g}$ filaments as the cutoff values ("up-and-down" method). The rats were placed in a plastic box $(20 \times 25 \times 15 \mathrm{~cm})$ on a metal mesh floor and allowed for 20-minutes adaptation. The filaments were then presented, in ascending order of strength $(0.16,0.4,0.6,1,1.4,2,4,6,8$, and $15 \mathrm{~g})$, and held for 6-8s perpendicular to the plantar surface with sufficient force to cause slight bending against the paw. Paw flinching or quick withdrawal were considered positive responses to pain. The paw-withdrawal threshold (PWT) was determined by consecutively increasing and decreasing the magnitude of the stimulus ("up-and-down" method). The nonparametric method of Dixon as previously described [11] was used to analyze the data. In the PSL model, withdrawal thresholds were measured in the animal using only the ipsilateral (ligated) paw. Mechanical allodynia and the effects of the drugs were assessed through the paw withdrawal threshold every 48 hours for 14 days after the partial sciatic nerve ligation. At the end of the experiment, rats were euthanized and excised sciatic nerve collected from the right hind limb located at the upper-thigh level and snap-frozen in liquid nitrogen for further investigation.

\section{Statistical Analysis}

All statistical analyses were carried out using the GraphPad Prism version 5.01 (GraphPad Software, La Jolla, CA, US). All values are depicted as a mean \pm SD and considered significant if $P<0.05$. Two-tailed Student's t-test was used to make statistical comparisons between two groups and two-way ANOVA used for comparisons of three or more groups.

\section{Results}

\section{BmK AGAP added to lidocaine in sciatic nerve block increases the intensity of sensory block.}

To investigate the analgesic effects of scorpion venom peptide, BmK AGAP as an adjuvant to lidocaine in sciatic nerve block, we performed partial sciatic nerve ligation (PSL) in the right hind limb of rats to induce a rapid onset and long-lasting mechanical allodynia. We first confirmed the presence of mechanical allodynia in all rats from the control group (received $0.9 \%$ saline), lidocaine group (received lidocaine and saline), and BmK AGAP groups (received lidocaine and BmK AGAP 2 or $1 \mathrm{mg} / \mathrm{kg}$ ) on the day 2 after the PSL procedure. No mechanical allodynia was recorded in the sham group (operated). An equal volume of $0.9 \%$ saline, lidocaine + saline, lidocaine + BmK AGAP $(1 \mathrm{mg} / \mathrm{kg})$ or lidocaine + BmK AGAP ( $2 \mathrm{mg} / \mathrm{kg}$ ) was injected into the upper thigh of the ligated right hind to cause sensory block. The von Frey filaments were then used to assess mechanical allodynia 30 minutes after the drugs solutions were injected. Two rats from the lidocaine group and three from the BmK AGAP groups exhibited 
complete motor blockade and were excluded from the analysis. Data from 10 rats from each group were analyzed.

BmK AGAP as an adjuvant to lidocaine increased the PWT in mechanical allodynia. We observed a significant increase in PWT in rats that were injected with BmK AGAP compared with rats that received lidocaine alone. The data showed that rats from the lidocaine group exhibited an increased PWT of $12.51 \mathrm{~g}$ compared with the baseline PWT of $0.90 \mathrm{~g}$. The rats from BmK AGAP $(1 \mathrm{mg} / \mathrm{kg})$ group exhibited an increased PWT of $13.67 \mathrm{~g}$ compared with the baseline PWT of $1.04 \mathrm{~g}$, whereas the rats from BmK AGAP $(2 \mathrm{mg} / \mathrm{kg})$ group exhibited an increased PWT of $14.78 \mathrm{~g}$ compared with baseline PWT of $1.00 \mathrm{~g}$ (Figure 1, Table 1). The data showed a significant $(P<0.0001)$ increase PWT of rats from BmK AGAP (1 $\mathrm{mg} / \mathrm{kg}$ ) group compared with rats from the lidocaine alone group. Similarly, rats that were injected with BmK AGAP $(2 \mathrm{mg} / \mathrm{kg})$ showed a significant $(P<0.001)$ increase in PWT compared with rats from the lidocaine alone group (Figure 1, Table 1). When we compared the PWT by the two different concentrations of BmK AGAP injected, we realized a significant $(P<0.0001)$ increased PWT in rats that were injected with BmK AGAP (2 mg/kg) compared with rats that received BmK AGAP (1 mg/kg). This evidence, therefore, suggests for the first time that BmK AGAP as an adjuvant to lidocaine in sciatic nerve block causes dose-dependent increase intensity of sensory block.

\section{BmK AGAP as an adjuvant to lidocaine causes dosedependent increase duration of analgesia.}

To examine the effects of BmK AGAP added to lidocaine on the duration of analgesia, we injected saline, lidocaine, lidocaine + BmK AGAP (1 mg/kg), or lidocaine + BmK AGAP (2 mg/kg) into the upper-thigh level of the ligated right hind to induce sensory blockade. The von Frey filaments were used to assess mechanical allodynia in the rats every 30 minutes interval until complete resolution of anesthesia (mechanical allodynia return to baseline). The total time (minutes) interval between the administration of drugs solutions and complete resolution of anesthesia was recorded as the duration of analgesia.

There was a prolonged duration of increased paw withdrawal threshold in rats that were supplemented with BmK AGAP compared with rats that received lidocaine injection alone (Figure 2; Figure 3 A, B). When we compared the effects of the two different concentrations of BmK AGAP, we realized a significantly prolonged duration of increased paw withdrawal threshold in rats that were supplemented with $\mathrm{BmK}$ $\operatorname{AGAP}(2 \mathrm{mg} / \mathrm{kg})$ compared with rats that received BmK AGAP (1 mg/kg) (Figure $3 \mathrm{C}$ ).

We observed for signs of adverse effects and realized that there were no episodes of any adverse effect during 24 hours of post-treatment period. These findings, therefore, suggest that scorpion venom peptide, BmK AGAP as an adjuvant to lidocaine in sciatic nerve block causes dose-dependent increase duration of analgesia without significant side effect.

\section{Discussion}

The current study was designed to test the hypothesis that scorpion venom peptide, BmK AGAP as an adjuvant to lidocaine in sciatic nerve block prolong the duration of analgesia. At the end of the study, two 
important observations emerged; first, the study results indicated that BmK AGAP as an adjuvant to lidocaine caused a dose-dependent increase intensity of sensory block (Figure 1, Table 1), and second, BmK AGAP as an adjuvant to lidocaine increased the duration of analgesia without significant adverse effects (Figure 2; Figure 3) .

In clinical practice, the choice of local anesthetic solution for peripheral nerve blocks depends on the speed of onset, the duration of action, the ability to induce a differential sensory nerve block and the potential for systemic toxicity $[1,2]$. The potency of local anesthetics nerve blocking increase with increasing molecular weight and lipid solubility [12]. Lidocaine is the most used local anesthetic for nerve blocks in the clinical setting due to its rapid onset, potency and tissues penetration. Lidocaine with epinephrine is the gold standard against which other local anesthetics are judged. A small volume of more concentrated local anesthetics increases the intensity and duration of nerve block compared with the more considerable amount of less concentrated local anesthetics. During a peripheral nerve block, a reasonably large quantity of local anesthetics is classically injected to generate a concentration gradient of local anesthetic into the nerve. This local anesthetic end up in the systemic circulation, and the free share, not bound to proteins or red blood cells, determine whether there will be systemic toxicity or not [13]. Adjuvants such as epinephrine, morphine, and fentanyl have long been added to local anesthetic to decrease systemic absorption and to prolong perioperative analgesia. With the introduction of several adjuvants, local anesthetic systemic toxicity has become rarer. However, reports indicate that some of these adjuvants prolong motor and sensory blocks, induce dose-dependent purities, urinary retentions and nausea [14]. The findings of this study showed BmK AGAP as an adjuvant to lidocaine in sciatic nerve block to increase and prolong the duration of analgesia without significant adverse effects. This evidence suggests BmK AGAP to be a potent analgesic adjuvant and its used in anesthesia may optimize perioperative analgesia without compromising anesthetic reliability.

Existing new developments to minimize local anesthetic systemic toxicity while optimizing perioperative analgesia include; the novel sustained release formulations, targeting of nociceptive-specific ion channels and targeting of nerve fibers subtypes [15]. BmK AGAP belongs to the group of long-chain scorpion toxin and has a molecular mass of 7142.6Da and 66 amino acids residues [4]. Animal studies have demonstrated that the scorpion venom peptide BmK AGAP exhibit strong analgesic activity with minimum advert effects compared with morphine $[4,5,6]$. However, it is not clear the mechanisms of the BmK AGAP analgesic activities, as well as the structure-function relationship of voltage-gated sodium channels. Evidence shows that it is a sodium channel-specific neurotoxin. It blocks neuron transmission by binding to voltage-independently at site- 3 of sodium channels and inactivate the activated sodium channels to elicit potent analgesic activity [4,5]. Local anesthetics such as lidocaine is indicated to mediate the analgesic activities of $\mathrm{Nav} 1.3, \mathrm{Nav} 1.7, \mathrm{Nav} 1.8$, and Nav 1.9 subunits by non-selectively blocking the voltage-gated sodium channels [16]. Neurotoxins have high affinity and more specificity for voltage-gated sodium channel, including Nav 1.7 and lower affinity for the cardiac voltage-gated sodium channel, Nav 1.5. This evidence endows neurotoxins with high potency for nerve block and no cardiotoxicity $[15,17]$. Study reports show that BmK AGAP modulates the inactivation behavior of Nav 1.7 when binding to the voltage-gated sodium channel [18]. Hence, addition of BmK AGAP to lidocaine in 
sciatic nerve blockade in this study caused dose-dependent increased intensity of sensory block and prolonged duration of analgesia.

As a preliminary investigation of BmK AGAP added to lidocaine in sciatic nerve block, the study presented some limitations. These limitations were related to the small sample size and the short term of the experiment. Further investigations should be performed on a longer period of treatment involving a larger sample size of rats.

\section{Conclusion}

In conclusion, BmK AGAP as an adjuvant to lidocaine enhances sensory block and prolong the duration of analgesia without significant adverse effects. The findings of this study suggest for the first time the use of scorpion venom peptide, BmK AGAP as an analgesic adjuvant in anesthesia to optimize perioperative analgesia.

\section{Abbreviations}

BmK AGAP- Buthus martensii Kasch Analgesic-Antitumor Peptide

PSL- Partial Sciatic Nerve Ligation

PWT- Paw Withdrawal Threshold

\section{Declarations}

\section{Ethics approval and consent to participate}

The Dalian Medical University, animal ethics committee, approved all the experimental procedures and were by the declaration of the national institutes of health guide for the care and use of laboratory animals.

\section{Consent to publish}

Not applicable

\section{Availability of data and materials}

All datasets use and analyzed during the present study are available from the corresponding author on reasonable request.

\section{Competing interests}

Authors declare that they have no competing interests 


\section{Funding}

This study was supported by the Liaoning Natural Science Foundation (Grant NO: 20170540290).

\section{Authors' contributions}

SK and QPW conceived and designed the study. QPW was responsible for the supervision and coordination of this study. SK and TZ performed most of the experiments. SK led the data analysis with inputs from QPW and TZ. SK wrote the first draft of the manuscript, and then QPW and TZ contributed to revising and reviewing the manuscript. All authors read and approved the final manuscript before submission.

\section{Acknowledgments}

We thank the head (Prof. Qiu Yan) and the staffs of the department of biochemistry, Dalian Medical University for making available all the necessary materials needed for this study.

\section{References}

[1] Warren D. T., Liu S. S. (2007). Analgesic adjuvants in Neuraxial Anesthesia: In Hadzic A. Textbook of Regional Anesthesia and Acute Pain Management. New York, McGraw-Hill. 133-143.

[2] McCartney C. J. L. (2007). Analgesic adjuvants in the peripheral nervous system: In Hadzic A. Textbook of Regional Anesthesia and Acute Pain Management. New York, McGraw-Hill. 145-155.

[3] Wang Q. Z., Zhang J. H., Tang L. (1994). Isolation, purification and a study on the analgesic effect of the analgesic peptide from scorpion venom of Buthus martensii Karsch. J Shenyang Coll Pharm. 11, $273-7$.

[4] Liu Y-F., Ma R-L., Wang S-L., Duan Z-Y., Zhang J-H., Wu L-J., Wu C-F. (2003). Expression of an antitumor-analgesic peptide from the venom of Chinese scorpion Buthus martensii Karsch in Escherichia coli. Protein Expression and Purification. 27, 253-258

[5] Li C-L., Liu X-F., Li G-X., Ban M-q., Chen J-Z., Qui Y., Zhang J-H., Wu C-F. (2016). Antinociceptive Effects of AGAP, a Recombinant Neurotoxic polypeptide: Possible Involvement of the Tetrodotoxin-Resistant Sodium Channels in Small Dorsal Root Ganglia Neurons. Front. Pharmacol. 7: 496.

[6] Ruan J-P., Mao Q-H., Lu W-G., Cai X-F., Chen J., Li Q., Fu Q., Yan H-J., Cao J-L., Cao P. (2018) Inhibition of spinal MARPKs by scorpion venom peptide BmK AGAP produces a sensory-specific analgesic effect. Molecular Pain. 14: 1-11

[7] ] Ma R., Cui Y., Zhou Y., Bao Y-M., Yang W-Y., Liu Y-F., Wu C-F., Zhang J-H. (2010). Location of the analgesic domain of scorpion toxin BmK AGAP by mutagenesis of disulfide bridges. Biochemical and Biophysical Research Communications. 394, 330-334. 
[8] Seltzer Z., Dubner R., and Shir Y. (1990). A novel behavioral model of neuropathic pain disorders produced in rats by partial sciatic nerve injury. Pain. 43, 205-218.

[9] Bennett, G. J., Chung, J. M., Honore, M., and Seltzer, Z. (2003) Models of neuropathic pain in the rat, Curr Protoc Neurosci. Chapter 9, Unit 9, 14.

[10] Ross A. Rat and mouse anesthesia and analgesia formulary and general drug information [Approved 2016, March $2^{\text {nd }}$ Available from https://docplayer.net/29603522-Rat-and-mouse-anesthesia-andanalgesia-formulary-and-general-drug-information.html

[11] Chaplan S. R., Bach F., Pogrel J., Chung J. M., Yaksh T. L. (1994). Quantitative assessment of tactile allodynia in the rat paw. $J$ Neurosci Methods 53: 55-63.

[12] Elliott C. E., Thomas L. C., Santos A. C. (2007) Newer Amide Local Anesthetics and Sustained-Release Local Anesthetics: In Hadzic A. Textbook of Regional Anesthesia and Acute Pain Management: New York, McGraw-Hill. 121-132.

[13] Lirk P., Picardi S., Hollmann M. W. (2014). Local anesthetics: 10 essentials. Eur. J. Anaesthesiol. 31: 575-585

[14] Yang Y., Yu L-Y., Zhang W-S. (2018). Clonidine versus other adjuncts added to local anesthetics for pediatric neuraxial blocks: a systematic review and meter analysis. Journal of Pain Research. 11: 10271037

[15] Lirk P., Hollmann M. W., Strichartz G. (2017). The science of Local Anesthesia: Basic Research, Clinical Application, and Future Directions. Anesthesia-Analgesia. DOI: 10.1213/ANE0000000000002665

[16] Mashour G. A., Lydic R. (7 September 2011). Neuroscientific Foundations of Anesthesiology. Oxford University Press. p. 154

[17] Kohane D. S., Smith S. E., Louis D. N., Colombo G., Ghoroghchian P., Hunfeld N. G. M., Berde C. B., Langer R. S. (2003). Prolonged duration local anesthesia from tetrodotoxin-enhance local anesthetic microspheres. Pain. 104: 415-421.

[18] Zhao Y., Cai X., Ye T., Huo J., Liu C., Zhang S., Cao Peng. (2011). Analgesic-Antitumor Peptide Inhibits Proliferation and Migration of SHG-44 Human Malignant Glioma Cells. Journal of Cellular Biochemistry. $112,2424-2434$.

\section{Tables}

See the Supplementary Files section to access Table 1.

\section{Figures}




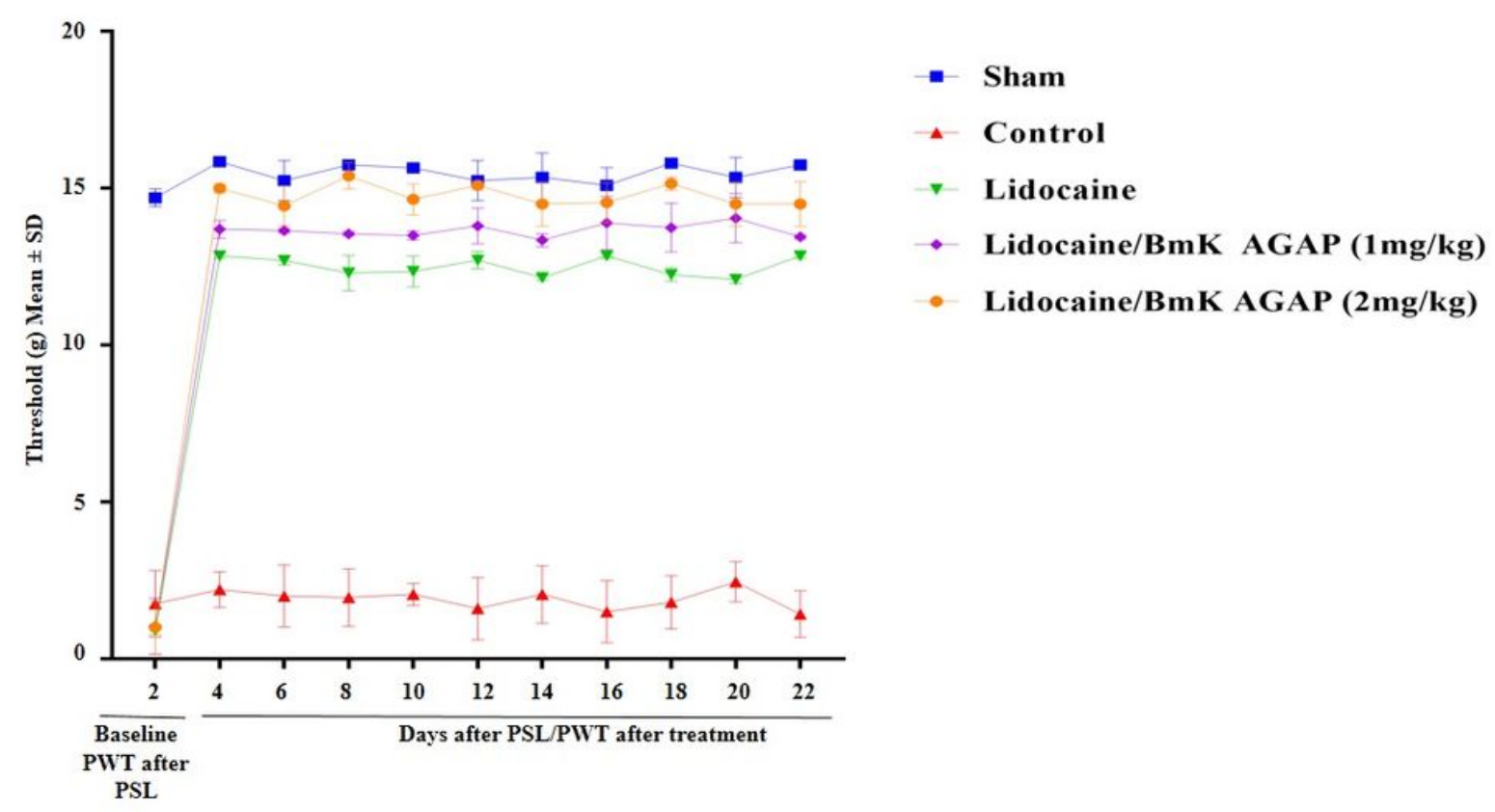

\section{Figure 1}

BmK AGAP as an adjuvant to lidocaine causes a dose-dependent increase in PWT when compared to lidocaine administered alone. The graph indicates the PWT values of the baseline taken two days after PSL and every 48 hours' time-points after sciatic nerve block. PWT = paw withdrawal threshold; PSL = partial sciatic nerve ligation. 


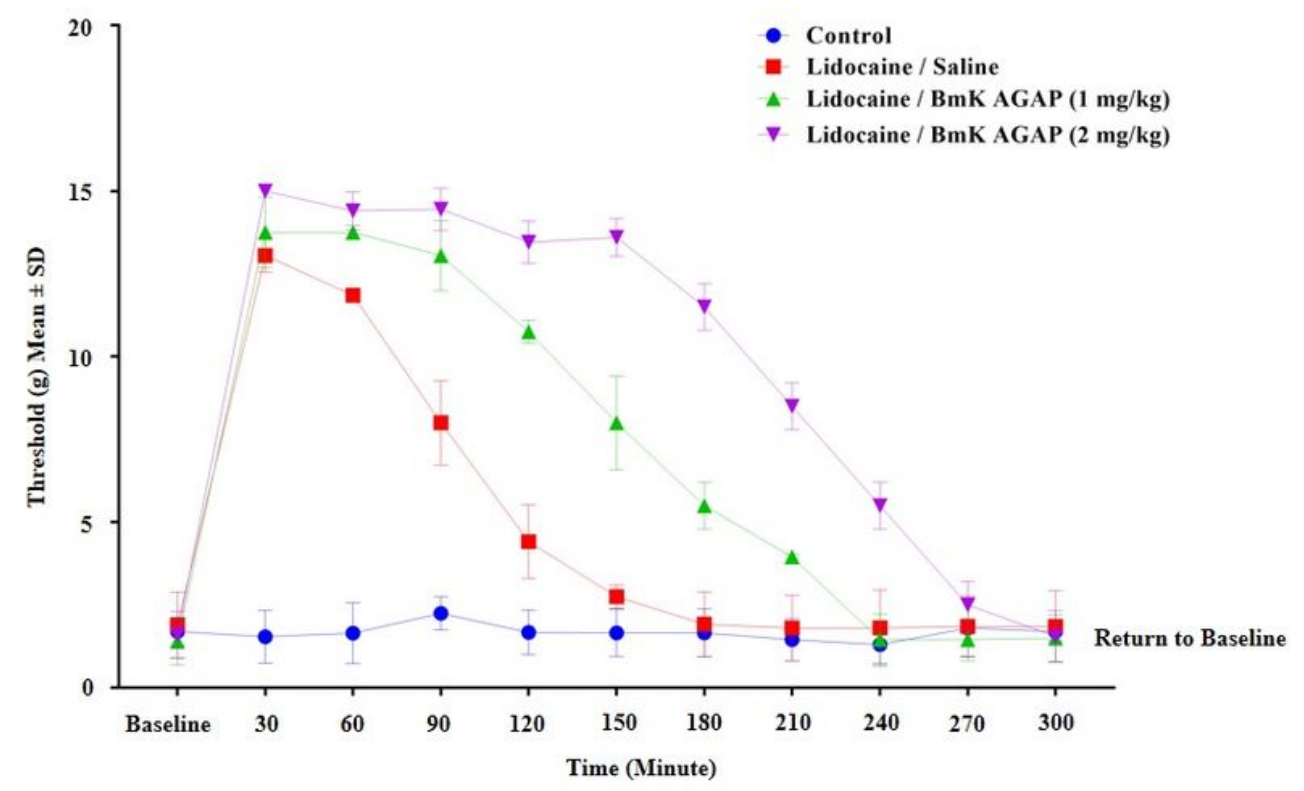

Figure 2

Duration of analgesia in a rat model of sciatic nerve block. BmK AGAP as an adjuvant to lidocaine in sciatic nerve block prolonged the duration of increased paw withdrawal threshold in dose-dependent manner. 


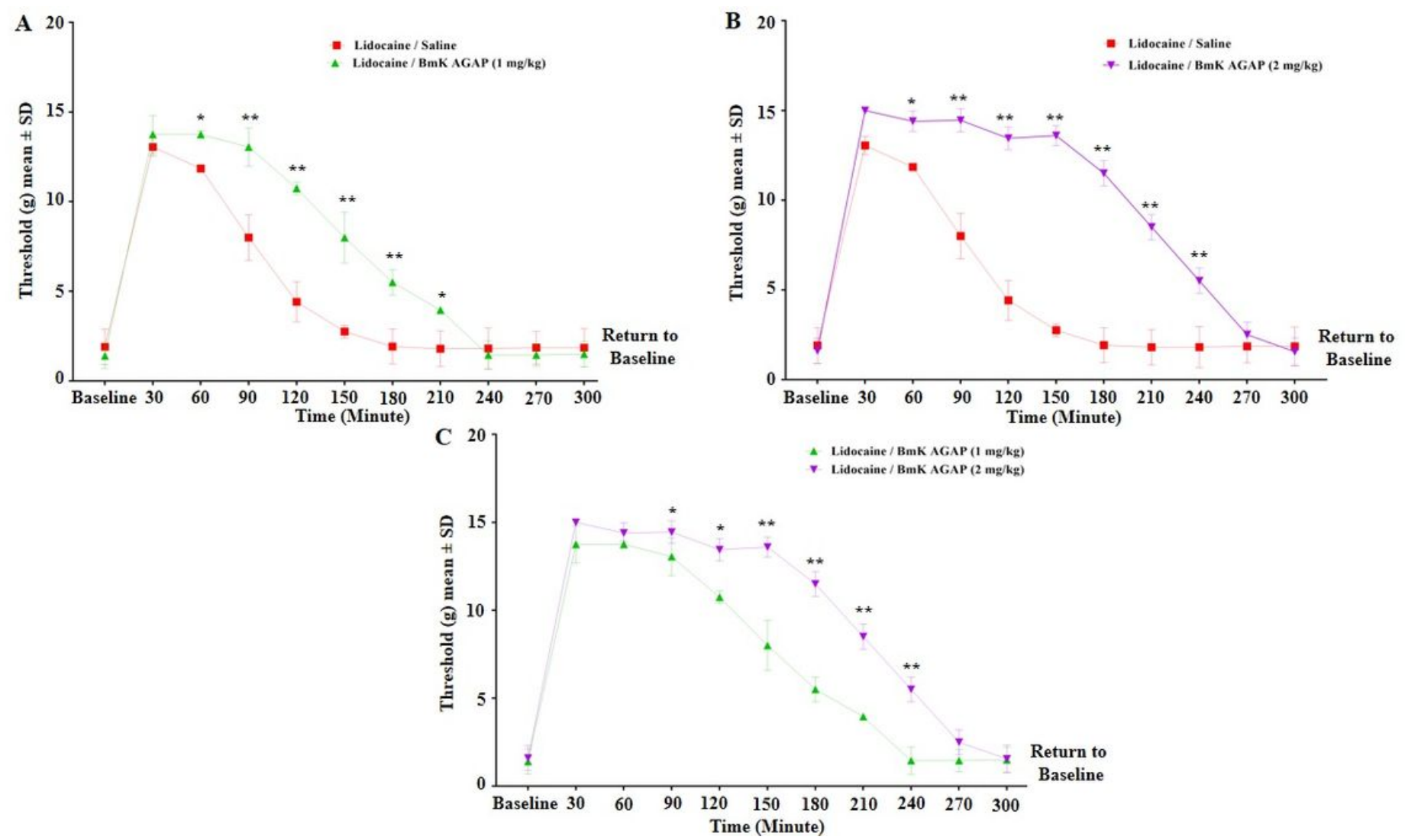

\section{Figure 3}

Increasing concentrations of BmK AGAP supplemented to lidocaine in sciatic nerve block prolonged the duration of increased paw withdrawal threshold to mechanical allodynia when compared with lidocaine alone. A, B, and C. Between-group comparisons from 60-240 minutes found multiple significant differences at separate time points.

\section{Supplementary Files}

This is a list of supplementary files associated with this preprint. Click to download.

- supplement1.pdf

- supplement2.tif 